Die Analyse lieferte folgende Werthe:

\begin{tabular}{|c|c|c|c|c|}
\hline \multirow{2}{*}{\multicolumn{2}{|c|}{ Theorie }} & \multicolumn{3}{|c|}{ Versuch } \\
\hline & & & II. & \\
\hline $\mathrm{C}$ & 51.61 & 51.26 & - & $\mathrm{pCt}$ \\
\hline $\mathrm{I}$ & 3.227 & 3.69 & - & 》 \\
\hline $\mathbf{T}$ & 15.05 & - & 15.34 & \\
\hline
\end{tabular}

Das Platinsalz stellt bräunlich-rothe, sehr schöne, mitunter grössere Krystalle dar. Es wurde aus heisser concentrirter Salzsäure umkrystallisirt und zeigte dann den Schmelzpunkt 253.5 . Bei $100^{\circ}$ getrocknet, hatte es folgenden Platingehalt:

Theorie
Pt 27.96
$28.09 \mathrm{pCt}$.

Der Gehalt an Platin des Isochinolins berechnet sich auf $29.12 \mathrm{pCt}$.

Die Base stellt also das $\gamma$-Methylisochinolin dar.

Aus $\underset{\mathrm{C}_{6} \mathrm{H}_{4}-\mathrm{CO}}{\mathrm{C}\left(\mathrm{CH}_{3}\right)_{2}-\mathrm{CO}} \mathrm{NH}$ resp. $\underset{\mathrm{C}_{6} \mathrm{H}_{4}-\mathrm{CO}}{\mathrm{C}\left(\mathrm{CH}_{3}\right)_{2}-\mathrm{CO}} \mathrm{N}\left(\mathrm{CH}_{3}\right)$ ist entstanden<smiles>CC(C)(C)/C=C/c1ccccc1</smiles>

430. E. Heim: Ueber die Einwirkung von Schwefelammon auf einige aromatische Dinitrokörper.

(Eingegangen am 12. Juli.)

Vor einiger Zeit ${ }^{1}$ ) habe ich die Einwirkung von Brombinitrobenzol (Schmp. $72^{\circ}$ ) auf $\beta$-Naphtylamin Leschrieben und gezeigt, dass in dem hierbei entstehenden $o-p$-Dinitrophenyl- $\beta$-naphtylamin durch Behandlung mit alkoholischem Schwefelammon die in Orthostellung zur Imidgruppe befindliche Nitrogruppe reducirt wird. Die Ausfübrung derselben Versuche mit dem $a$-Naphtylamin führte zu ganz analogen Resultaten.

Dinitrophenyl- $\alpha$-naphtylamin.

Entsteht beim Erhitzen von Brombinitrobenzol ( 1 Mol.) and a-Naphtylamin (1 Mol.) in alkoholischer Lösung im Druckrohr auf

1) Diese Berichte XXI, 589.

Berichte d. D. chem. Gesellschalt. Jahro. XXI. 
$120^{\circ}$ and beim Kochen der alkoholischen Lösung von Brombinitrobenzol (1 Mol.) und $\alpha$-Naphtylamin (2 Mol.).

$10 \mathrm{~g}$ (1 Mol.) Brombinitrobenzol (Schmp. $72^{\circ}$ ) wurden in $40 \mathrm{ccm}$ Alkohol gelöst und in die warme Lösung $11.6 \mathrm{~g}$ (2 Mol.) a-Naphtylamin eingetragen, welche unter Rothfärbung alsbald in Lösung gingen. Die Flüssigkeit wurde darauf 3 Stunden am Rückflusskühler gekocht. Schon nach kurzer Zeit begann die Abscheidung einer orangerothen Krystallmasse, welche nach dem Erkalten abgesaugt und zur Entfernung des etwa noch vorhandenen $\alpha$-Naphtylamins und vor allem des reichlich gebildeten Bromhydrats desselben gründlich mit heissem Wasser, dann mit Salzsäure und zum Schluss wieder mit Wasser ausgewaschen wurde. Die Mutterlauge wurde durch Einengen zu nochmaliger Krystallisation gebracht and das abgeschiedene Product in gleicher Weise gereinigt. Die Ausbeute der so erhaltenen schon ziemlich reinen Substanz betrug $8 \mathrm{~g}$.

Durch einmalige Krystallisation aus Eisessig oder absolutem Alkohol wurde dieselbe in prachtvoll glänzenden, orangerothen Nadeln vom Schmelzpunkt $190.5^{\circ}$ analysenrein erhalten.

Berechnet

$\begin{array}{lc}\text { für } \mathrm{C}_{16} \mathrm{H}_{11} \mathrm{~N}_{3} \mathrm{O}_{4} \\ \mathrm{C} & 62.14 \\ \mathrm{H} & 3.56 \\ \mathrm{~N} & \mathbf{1 3 . 5 9} \\ \mathrm{O} & 20.71\end{array}$

Gefunden

\begin{tabular}{|c|c|c|c|}
\hline I. & II. & III. & IV. \\
\hline 62.40 & 61.94 & - & - \\
\hline 401 & 3.93 & - & - \\
\hline- & - & 13.68 & 13.51 \\
\hline & - & - & - \\
\hline
\end{tabular}

Die Entstehungsgleichung und Constitutionsformel des Körpers sind ganz analog den für das entsprechende Dinitrophenyl- $\beta$-naphtylamin (a. a. O.) angegebenen.

Das o-p-Dinitrophenyl-a-naphtylamin ist in Wasser unlöslich, in Benzol, Toluol, Aceton, Chloroform sehr leicht löslicb; in Alkohol und Eisessig löst es sich leicht in der Siedhitze, dagegen sehr wenig bei gewöhnlicher Temperatur.

Alkoholisches Kali löst mit dunkelrother, concentrirte Schwefelsäure mit tiefdunkelblauer Farbe, welche durch Erhitzen nicht verändert wird.

Durch alkoholisches Schwefelammon wird eine der beiden Nitrogruppen reducirt.

Nitroamidophenyl-a-naphtylamin.

$5 \mathrm{~g}$ Dinitrophenyl- $\alpha$-naphtylamin wurden, möglichst fein verrieben, in einem Kolben mit $750 \mathrm{ccm}$ Alkohol übergossen und $75 \mathrm{ccm}$ käufliches gelbes Schwefelammon binzugefügt. Nachdem der Kolbeninhalt 2 Tage lang verkorkt bei gewöhnlicher Temperatur unter häufigem Umschütteln aufbewahrt war, wurde er 2 Stunden lang auf dem Wasserbade bei gelinder Wärme erbalten, die schliesslich zum Aufkochen gesteigert wurde. Aus der so erhaltenen klaren dunkel- 
braunen Lösung fiel beim Erkalten der frei gewordene Schwefel aus. Das Filrat von demselben wurde bis auf $1 / 5$ seines Volums abdestillirt und schied dann den Nitroamidokörper als dunkelgelben krystallinischen Niederschlag ab. Dieser wurde mit Wasser, dann mit Schwefelkohlenstoff gewaschen und schliesslich mehrmals aus 50 procentigem Alkohol oder ebenso starker Essigsäure umkrystallisirt. Man erhält so dunkelgelbe derbe Nadeln vom Schmelzpunkt $145-147^{\circ}$. Die Ausbeute an reiner Substanz betrug $60 \mathrm{pCt}$. der Menge des angewandten Dinitrokörpers.

\begin{tabular}{ccccc}
\multicolumn{2}{c}{ Berechnet } & \multicolumn{2}{c}{ Gefunden } \\
für $\mathrm{C}_{16} \mathrm{H}_{13} \mathrm{~N}_{3} \mathrm{O}_{2}$ & 1. & II. \\
$\mathrm{C}$ & 68.82 & 68.87 & - & $\mathrm{pCt}$. \\
$\mathrm{H}$ & 4.66 & 5.02 & - & $\$$ \\
$\mathrm{~N}$ & 15.05 & - & 15.28 & \\
$\mathrm{O}$ & 11.47 & - & - &
\end{tabular}

Der Körper löst sich schwer in heissem Wasser, sehr leicht in Alkohol, Eisessig, Aceton, Chloroform, warmem Benzol und Toluol, mässig in Aether. Aus allen diesen Lösungsmitteln krystallisirt er jedoch sehr schlecht.

Concentrirte Schwefelsäure giebt eine anfangs dunkelgrüne, bald dunkelblaue Lösung, aus welcher Wasser rothbraune Flocken fällt.

Die Einwirkung von salpetriger Säure bewies, dass die durch Behandlung mit Schwefelammon erzeugte Amidogruppe in Orthostellung zur Imidgruppe steht. Es entstand nämlich hierbei:

Nitroazoïmidophenyl-a-naphtylamin.

$2 \mathrm{~g}$ Nitroamidophenyl- $x$-naphtylamin wurden in $50 \mathrm{ccm}$ Alkohol gelöst, mit Salzsäure angesäuert und mit $10 \mathrm{ccm}$ einer 25 procentigen alkoholischen Lösung von Aethylnitrit versetzt. Alsbald begann die Abscheidung einer schweren krystallinischen gelben Fällung. Nach einer Viertelstunde wurde das Reactionsgemisch kurze Zeit anf dem Wasserbade gekocht. Die Menge der abgeschiedenen Substanz war gleich der des angewandten Nitroamidokörpers. Dieselbe* wurde aus absolutem Alkohol, in welchem sie in der Kälte fast garnicht, in der Hitze ziemlich schwer löslich ist, in zimmtbraunen, glänzenden, verwachsenen Kryställehen erhalten. Der Schmelzpunkt liegt bei $182^{\circ}$.

Die Analyse ergab Zahlen, welche den Körper als Azoümidoderivat der vorstehend beschriebenen Substanz von der Formel:

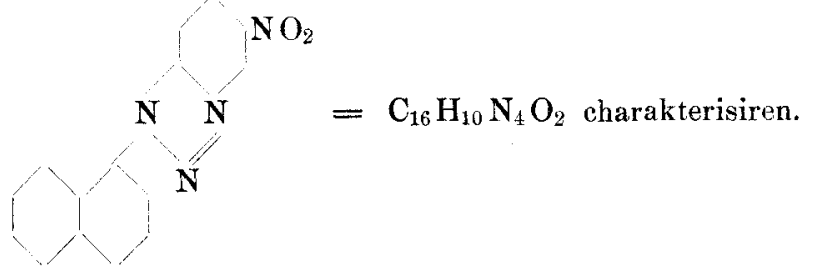




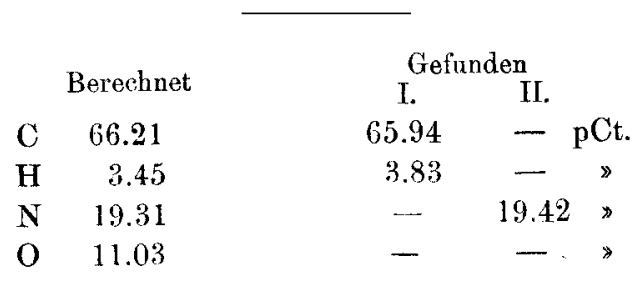

Der Körper ist unlöslich in Wasser, schwer löslich in Aether und Schwefelkohlenstoff, leicht löslich in Aceton, Chloroform, ziemlich löslich in Benzol, Toluol und heissem Eisessig.

Concentrirte Schwefelsäure giebt eine dunkelgrüne Lösung, aus welcher Wasser die unveränderte Substanz abscheidet. Ebenso fällt aus der heissen Lösung in alkoholischem Kali die Azoïmidoverbindung beim Erkalten unverändert.

\section{Nitroortho phenylendiamin.}

Die Einwirkung von Schwefelammon auf die Muttersubstanz der Dinitrophenylnaphtylamine, das $o-p$-Dinitranilin, ist scbon im Jahre 1853 durch Gottlieb ${ }^{1}$ ) untersucht worden, welcher ein in dunkelrothen Nadeln krystallisirendes Nitrophenylendiamin erhielt. A. W.Hof$\operatorname{mann}{ }^{2}$ ) unterwarf dasselbe im Jahre 1860 der Einwirkung von salpetriger Säure und gewann so eine gut krystallisirende Substanz, die durch Analysenergebniss und Eigenschaften als Nitroazoïmidophenylendiamin charakterisirt wurde. Obgleich dadurch nachgewiesen war, dass in dem Dinitranilin durch Schwefelammon die in Orthostellung zur Amidgruppe befindliche Nitrogruppe reducirt wird, und der Gottlieb'sche Körper demnach als $p$-Nitro orthophenylendiamin aufzufassen ist, so haben doch später Biedermann und Ledouxi3) denselben mit Bestimmtheit als Nitroparaphenylendiamin hingestellt, indem sie jhn für identisch mit einem von ihnen aus Derivaten des Paraphenylendiamins erhaltenen Product erklärten. Ladenburg ${ }^{4}$ ) hat dann diesen Widersprueh aufgeklärt, indem er nachwies, dass Biedermann und Ledoux durch Kochen des Nitrodiacetylparaphenylendiamins mit Natronlauge nicht; wie sie glaubten, Nitroparaphenylendiamin, sondern ein Nitromonoacetylparaphenylendiamin erhalten hatten, dessen Schmelzpunkt er übrigens auch nicht, wie jene Forscher angegeben hatten, mit dem des Gottlieb'schen Körpers übereinstimmend fand. Ladenburg's Versuch, durch trockene Destillation der bei Einwirkung von Essigsäureanhydrid auf das Gottlieb'sehe Nitrophenylendiamin ent-

\footnotetext{
1) Ann. Chem. Pharm. 85, 27.

2) Ann. Chem. Pharm. 115, 249.

3) Diese Berichte VII, 1531.

4) Diese Berichte XVII, 147.
} 
stehenden Diacetylverbindung eine Aethenylanhydrobase zu erhalten und damit einen weiteren Beweis für die Orthostellung der beiden Amidogruppen zu erbringen, scheiterte an Verkohlung. Dagegen gewann er das Nitroparaphenylendiamin durch Kochen seiner Diacetylverbindung mit concentrirter Salzsäure und zeigte, dass es von dem von Gottlieb erhaltenen Isomeren ganz verschiedene Eigenschaften besitzt.

Ein weiteres Mittel, die Orthostellung der beiden Amidgruppen nachzuweisen, bot sich in der Reaction zwischen Orthodiaminen und Orthodiketonen, welche, wie Witt in seinen Arbeiten über die Eurhodine gezeigt hat, zu ganz besonders schönen und charakteristischen Verbindungen führt. Ich habe daher die Einwirkung von Phenantrenchinon auf den Gottlieb'schen Körper untersucht, in der Erwartung, so zu einem nitrirten Azinderivat zu gelangen, welches sich durch geeignete Reductionsmittel in einen der Klasse der Eurhodine angehörigen Körper überführen lassen würde.

Für die Darstellung des $p$-Nitro-o-phenylendiamins stellte sich folgendes Verfahren als das zweckmässigste heraus:

5 g Dinitranilin vom Schmelzpunkt $175^{\circ}$, dargestellt aus Brom-

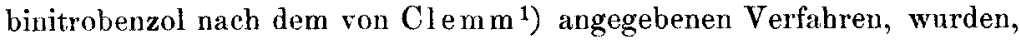
fein gepulvert, in einem Kolben mit $250 \mathrm{ccm}$ Alkohol und $50 \mathrm{ccm}$ käuflichen gelben Schwefelammons übergossen und verkorkt stehen gelassen. Nach einigen Tagen war alles Dinitranilin verschwunden, dagegen hatte sich ein Theil des Reactionsproductes bereits in Gestalt dunkelrother Nadeln abgeschieden. Das Gemisch wurde darauf kurze Zeit aufdem Wasserbade gekocht, vom abgeschiedenen Schwefel abfiltrirt und der Alkohol bis auf die Hälfte seines Volumes abdestillirt. Beim Erkalten schied sich eine dunkelrothe Krystallmasse ab, von welcher durch Einengen des Filtrats mehr gewonnen werden konnte. Nach dem Waschen mit Schwefelkohlenstoff und mit wenig kaltem Wasser wurde die Substanz durch Umkrystallisiren völlig gereinigt. Besonders schöne Krystalle lieferte bei ganz allmählicher Abkühlung eine heiss gesättigte Lösung in Toluol, in dem der Körper bei gewöhnlicher Temperatur fast unlöslich, in der Siedhitze auch nur schwer löslich ist.

Es wurden so prachtvoll glänzende dunkelrothe Blättehen erhalten, welche den schon von Gottlieb erwähnten, bläulichen Schiller in der Flüssigkeit, sowie den lichtrothen Schein in trockenem Zustande zeigten. Der Schmelzpunkt ist von Gottlieb und Ladenburg übereinstimmend bei $195^{\circ}$ angegeben. Ich fand denselben, durch weiteres Umkrystallisiren unabänderlich, bei $198^{\circ}$. Die Beobachtung desselben wird jedoch dadurch erschwert, dass die Substanz im Schmelzpunkts-

1) Journ. für prakt. Chem. [2] 1, 145. 
röhrchen schon bei $190^{\circ}$ zu sublimiren beginnt. Die Reinheit der Substanz bewies die Analyse.

\begin{tabular}{lcccc} 
& Berechnet & \multicolumn{3}{c}{ Gefunden } \\
& für $\mathrm{C}_{6} \mathrm{H}_{7} \mathrm{~N}_{3} \mathrm{O}_{2}$ & \multicolumn{1}{c}{$\mathrm{I}}$. & \multicolumn{1}{c}{ II. } \\
$\mathrm{C}$ & 47.06 & 47.18 & - & $\mathrm{pCt}$. \\
$\mathrm{H}$ & 4.58 & 4.82 & - & $\gg$ \\
$\mathrm{N}$ & 27.45 & - & 27.39 & $\gg$ \\
$\mathrm{O}$ & 20.92 & - & - & $\gg$
\end{tabular}

Nitrophenophenanthrazin.

Die zum Sieden erhitzte Lösung von $1 \mathrm{~g}$ Nitrophenylendiamin in $100 \mathrm{ecm}$ Eisessig wurde in die ebenfalls siedend heisse Lösung von $1.4 \mathrm{~g}$ Phenantrenchinon in $150 \mathrm{ccm}$ Eisessig eingegossen. Die Mischung blieb einige Secunden klar, um sich dann plötzlich in einen aus feinen citronengelben Nadeln bestehenden Krystallbrei zu verwandeln, welcher noch einige Minuten im Sieden erhalten wurde. Durch Absaugen der Mutterlauge wurden so $2 \mathrm{~g}$ des fast ganz reinen Productes erhalten. Zur Analyse wurde die Substanz durch Krystallisation aus Tolnol oder Eisessig völlig gereinigt.

\begin{tabular}{|c|c|c|c|c|c|}
\hline & Berechnet & & & & \\
\hline $\mathrm{fi \tilde { }}$ & $\mathrm{C}_{20} \mathrm{~B}_{11} \mathrm{~N}_{3} \mathrm{O}_{2}$ & I. & II. & IIII. & \\
\hline $\mathrm{C}$ & 73.85 & 74.07 & 73.60 & - & pCt. \\
\hline H & 3.39 & 3.75 & 3.75 & - & $\triangleright$ \\
\hline S & 12.92 & - & - & 12.83 & » \\
\hline & 9.85 & - & - & - & » \\
\hline
\end{tabular}

Der Schmelzpunkt liegt bei $251^{\circ}$. Bei höherer Temperatur sublimirt der Körper fast vollständig unzersetzt in feinen, wolligen, gelben Nadeln. Er ist unlöslich in Wasser, sehr sehwer löslich in Alkohol, Aether, Eisessig; ziemlich schwer löslich in Aceton, leicht löslich in Chloroform, siedendem Benzol und Tolnol.

Concentrirte Schwefelsäure liefert eine prachtvoll purpurrothe Lösung, die auf Wasserzusatz orangefarbig, durch mehr Wasser unter Abscheidung bellgelber Flocken entfärbt wird.

Bei Behandlung mit alkobolischem Schwefelammon unter Druck liefert der Körper das entsprechende Eurbodin.

\section{Amidophenophenanthrazin.}

$1 \mathrm{~g}$ Nitrophenophenantrazin wurde mit einem Ueberschuss einer mit Schwefelwasserstoff gesättigten 10 procentigen Lösung von Ammoniak in Alkohol 3 Stunden lang im Druckrohr auf $170^{\circ}$ erhitzt. Nach dem Erkalten batte sich ein schwerer, dunkelgelber, krystallinischer Niederschlag abgeschieden. Derselbe wurde zur Entfernung beigemengten Schwefels und Schwefelammonis mit Schwefelkohlenstoff 
und Wasser gewaschen. Seine Menge betrug $0.8 \mathrm{~g}$. Durch Umkrystallisiren aus Tolnol wurde er in eigelben, kurzen, dicken, warzenförmig gruppirten Nadeln erhalten. In allen Eigenschaften stimmt die Substanz mit dem von $W_{\text {itt }}{ }^{1}$ ) durch Einwirkung einer reducirten Lösung von Chrysoïdin auf Phenantrenchinon erhaltenen Eurhodin überein. Sie zeigt in prachtvoller Weise die grüngelbe Fluorescenz der ätherischen Lösung, sowie das charakteristiscbe Farbenspiel der Lösung in concentrirter Schwefelsäure. Der von Witt nicht angegebene Schmelzpunkt wurde bei $279^{\circ}$ gefunden. Zugleich beginnt die Substanz zu sublimiren. Auch der Stickstoffgehalt wurde entsprechend der Formel $\mathrm{C}_{20} \mathrm{H}_{13} \mathrm{~N}_{3}$ gefunden.

$$
\begin{array}{ccc} 
& \text { Berechnet } & \text { Gefunden } \\
N & 14.24 & 14.26 \mathrm{pCt} .
\end{array}
$$

Da sonach die Orthostellung der beiden Amidgruppen im Gottlieb'schen Nitrophenylendiamin ausser Zweifel war, schien es unwabrscheinlich, dass dasselbe unter keiner Bedingung im Stande sein sollte, mit Essigsäureanhydrid eine Anhydrobase zu bilden. Ich versuchte daher, durch Erhitzen unter Druck zu einer solchen zu gelangen.

Nitroäthenylorthophenylendiamin.

$1 \mathrm{~g}$ Nitro-o-Phenylendiamin wurde mit $8 \mathrm{~g}$ Essigsäureanhydrid im Druckrohr 4 Stunden lang auf $190^{\circ}$ erhitzt. Nach dem Erkalten wurde die entstandene dunkle Lösung mit verdünnter Schwefelsäure vermischt, zum Sieden erbitzt und von ungelöster kohliger Substanz abfiltrirt. Das Filtrat wurde dann mit Ammoniak übersättigt. Der entstandene Niederschlag wurde durch Krystallisation aus Wasser in gut ausgebildeten derben gelbbraunen Nadeln erhalten. Der Schmelzpunkt derselben liegt bei $216^{\circ}$, während der der von Ladenburg beschriebenen Diacetylverbindung in Uebereinstimmung mit den Augaben desselben bei $227^{\circ}$ gefunden wurde, übrigens mit Zersetz'ng verbunden ist.

Die erwähnten Krystalle enthalten Krystallwasser, dessen Menge sich nicht genau ermitteln liess, da die Substanz schon an der Luft verwittert. Durch Erhitzen auf $120^{\circ}$ lässt sich der Körper wasserfrei erhalten und zeigte den für eine Aethenylrerbindung von der $\mathrm{N}$

Formel $\left(\mathrm{NO}_{2}\right) \mathrm{C}_{6} \mathrm{H}_{3} \quad \mathrm{C} . \mathrm{CH}_{3}$ berechneten Stickstoffgehalt. $\mathrm{NH}$

$$
\begin{aligned}
& \text { Ber. für } \mathrm{C}_{8} \mathrm{H}_{7} \mathrm{~N}_{3} \mathrm{O} \\
& \text { Gefunden } \\
& \text { N } 23.73 \quad 23.97 \text { pCt. }
\end{aligned}
$$

1) Diese Berichte X[X, 445 . 
Die Substanz löst sich leicht in heissem Wasser, in Alkohol, Benzol, Toluol, Chloroform, ziemlich leicht in Aether. In verdünnten Säuren ist sie gleichfalls leicht löslich und wird durch Alkali aus: diesen Lösungen in Gestalt gelbbrauner Flocken gefällt.

Ich habe schliesslich die Einwirkung von Schwefelammon auf ein in Ortbo- und Parastellung zum Stickstoff nitrirtes tertiäres Amin untersucht, nämlich das o-p -Dinitrodimethylanilin vom Schmelzpunkt $87^{\circ}$, dessen Darstellung am besten nach der von Mertens ${ }^{1}$ ) gegebenen Vorschrift erfolgt. Auch hier reducirt, wie nachstehende Versuche zeigen, Schwefelammon in Orthostellung. Während aber in den bisher beschriebenen Fällen die Reaction stets sehr glatt und ohne Bildung erheblicher Mengen von Nebenproducten von Statten gegangen war, bildete sich hier stets vorwiegend ein schmieriges schwefelhaltiges Product, und es gelang nicht, die Ausbeute an dem Nitroamidokörper über $10 \mathrm{pCt}$. des Ausgangsmaterials zu steigern.

\section{Nitroamidodimethylanilin.}

$10 \mathrm{~g}$ Dinitrodimethylanilin wurden in $300 \mathrm{ccm}$ Alkohol gelöst und die warme Lösung mit $100 \mathrm{ccm}$ gelben Schwefelammons versetzt, wobei die Temperatur so regulirt wurde, dass die bei jedesmaligem Zusatz von Schwefelammon entstehende Fällung sich beim Umschütteln sogleich wieder in der dunkelbraunen Flüssigkeit auflöste. Diese wurde 2 Stunden lang bei geringer Wärme auf dem Wasserbade erhalten, dann kurze Zeit aufgekocht und nach dem Abfiltriren des abgeschiedenen Schwefels auf etwa den dritten Theil ihres Volums abdestillirt. Von dem hierbei abgeschiedenen schmierigen Product wurde abgegossen oder abfiltrirt und aus der Lauge durch Eindampfen. eine weitere Menge davon erhalten. Dasselbe erstarrt in der Regel bei längerem Liegen oder beim Einbringen eines Krystalls der Nitroamidoverhindung. Beim Auskochen mit Wasser bleibt der Schwefel und die grösste Menge der schmierigen Nebenproducte ungelöst, während aus der Lösung beim Erkalten sich braune derbe Nadeln abscheiden. Durch mehrfache Krystallisation aus viel siedendem Wasser erhält man orangegelbe Nadeln vom Schmelzpunkt $63^{\circ}$, welche das reine Reductionsproduct darstellen.

\begin{tabular}{|c|c|c|c|c|}
\hline \multicolumn{2}{|c|}{ Berechnet } & \multicolumn{3}{|c|}{ Gefunden } \\
\hline & $\mathrm{C}_{8} \mathrm{H}_{11} \mathrm{~N}_{3} \mathrm{O}_{2}$ & I. & II. & \\
\hline $\mathrm{C}$ & 53.04 & 52.88 & - & $\mathrm{pCt}$ \\
\hline $\mathrm{H}$ & 6.08 & 6.34 & - & » \\
\hline $\mathrm{N}$ & 23.20 & - & 23.19 & » \\
\hline $\mathrm{O}$ & 17.68 & - & - & 》 \\
\hline
\end{tabular}

1) Diese Berichte XIX, 2124. 
Der Körper ist schwer löslich in heissem, noch schwerer in kaltem Wasser; dagegen leicht löslich in Alkohol, Aether, Schwefelkohlenstoff, spielend in Benzol, Toluol, Aceton and Chloroform. Er löst sich leicht in verdünnten Mineralsäuren, ebenso in Essigsäure, aus deren Lösung er beim vorsichtigen Uebersättigen mit Ammoniak auskrystallisirt.

Er ist mit Wasser- und Alkoholdämpfen, wenn auch in geringer Menge flüchtig. Trocken lässt er sich gut aufbewahren, in feuchtem Zustande, sowie in wässeriger oder alkoholischer Lösung färbt er sich bald dunkel.

Beim Erhitzen mit alkoholischem Kali wird er nicht, wie die Dinitroverbindung, gespalten, sondern krystallisirt unverändert aus der dunkelrothen Lösung. Mit Essigsäureanbydrid liefert er eine krystallisirende Acetylverbindung.

Die Entscheidung der Stellungsfrage wurde durch Einwirkung von salpetriger Säure herbeigeführt.

\section{Paranitrodimethylanilin.}

$2 \mathrm{~g}$ Nitroamidodimethylanilin wurden in einem Kölbchen in $20 \mathrm{ccm}$ einer 25 procentigen alkoholischen Lösung von Aethylnitrit gelöst und mit $10 \mathrm{ccm} 25$ procentiger Salzsäure versetzt. Das Gemisch erwärmte sicb stark unter lebhafter Stickstoffentwickelung und deutlichem Aldebydgeruch. Als die Reaction nachliess, wurde erwärmt, bis die Gasentwickelung beendet war. Beim Erkalten krystallisirte ein Theil des Reactionsproductes in glänzenden strohgelben Nadeln mit bläulichem Schimmer aus, eine weitere Menge liess sich aus dem Filtrat hiervon durch Abdampfen oder Wasserzusatz erhalten.

Der erhaltene Körper zeigt den Schmelzpunkt $162^{\circ}$, der sich dureh Umkrystallisiren nicht ändert. Dies, sowie seine sonstigen Eigenschaften lassen keinen $Z$ weifel, dass es mit dem von Weber ${ }^{1}$ ) dureh Nitrirung von Dimethylanilin, von Leymann ${ }^{2}$ ) aus $p$-Nitroehlorbenzol und Trimethylamin erhaltenen $p$-Nitrodimethylanilin identisch ist.

Es geht hieraus hervor, dass die dureh Aethylnitrit eliminirte Amidgruppe sich in Orthostellung $\mathrm{zu}$ dem basischen Stickstoffatom befand, dass also die Amidirung durch Schwefelammon auch in den $o-p$-Dinitroderivaten tertiärer Phenylamine in der Orthostellung stattfindet.

Berlin, im Juli 1888.

1) Diese Berichte $X, 761$.

2) Diese Berichte XV, 1234. 